\title{
Development of Animal Models of Local Retinal Degeneration
}

\author{
Henri Lorach, ${ }^{1-3}$ Jennifer Kung, ${ }^{2}$ Corinne Beier ${ }^{4}$ Yossi Mandel, ${ }^{1,5}$ Roopa Dalal, ${ }^{2}$ Philip Huie, ${ }^{1,2}$ \\ Jenny Wang, ${ }^{1,2}$ Seungjun Lee, ${ }^{2}$ Alexander Sher, ${ }^{4}$ Bryan William Jones, ${ }^{6}$ and Daniel Palanker ${ }^{1,2}$
}

${ }^{1}$ Hansen Experimental Physics Laboratory, Stanford University, Stanford, California, United States

${ }^{2}$ Department of Ophthalmology, Stanford University, Stanford, California, United States

${ }^{3}$ Institut de la Vision, Paris, France

${ }^{4}$ Santa Cruz Institute for Particle Physics, University of California, Santa Cruz, California, United States

${ }^{5}$ Faculty of Life Sciences, Bar Ilan University, Ramat Gan, Israel

${ }^{6}$ Ophthalmology and Visual Sciences, Moran Eye Center, University of Utah, Salt Lake City, Utah, United States

Correspondence: Henri Lorach, Hensen Experimental Physics Laboratory, 452 Lomita Mall, Stanford, CA 94305, USA;

henri.lorach@gmail.com.

HL and JK contributed equally to the work presented here and should therefore be regarded as equivalent authors.

Submitted: November 5, 2014

Accepted: June 14, 2015

Citation: Lorach H, Kung J, Beier C, et al. Development of animal models of local retinal degeneration. Invest Ophthalmol Vis Sci. 2015;56:46444652. DOI:10.1167/iovs.14-16011
Purpose. Development of nongenetic animal models of local retinal degeneration is essential for studies of retinal pathologies, such as chronic retinal detachment or age-related macular degeneration. We present two different methods to induce a highly localized retinal degeneration with precise onset time, that can be applied to a broad range of species in laboratory use.

Methods. A 30- $\mu \mathrm{m}$ thin polymer sheet was implanted subretinally in wild-type (WT) rats. The effects of chronic retinal separation from the RPE were studied using histology and immunohistochemistry. Another approach is applicable to species with avascular retina, such as rabbits, where the photoreceptors and RPE were thermally ablated over large areas, using a high power scanning laser.

REsults. Photoreceptors above the subretinal implant in rats degenerated over time, with $80 \%$ of the outer nuclear layer disappearing within a month, and the rest by 3 months. Similar loss was obtained by selective photocoagulation with a scanning laser. Cells in the inner nuclear layer and ganglion cell layer were preserved in both cases. However, there were signs of rewiring and decrease in the size of the bipolar cell terminals in the damaged areas.

Conclusions. Both methods induce highly reproducible degeneration of photoreceptors over a defined area, with complete preservation of the inner retinal neurons during the 3-month follow-up. They provide a reliable platform for studies of local retinal degeneration and development of therapeutic strategies in a wide variety of species.

Keywords: geographic atrophy, animal model, AMD

\begin{abstract}
A s populations age, the prevalence of age-related macular Adegeneration (AMD) is rapidly increasing. Worldwide, it affects $8.7 \%$ of the population between 45 and 85 years of age. ${ }^{1}$ This pathology is characterized by progressive loss of central vision, while peripheral vision remains well preserved. Central vision rapidly deteriorates when the disease reaches the phase where either blood vessels from the choroid breach the RPE and the Bruch's membrane (wet form), or local atrophy of the pigment epithelium expands in the macula (dry form) leading to geographic atrophy. Since the introduction of anti-VEGF medications, wet forms of AMD can be effectively controlled, while the dry form, representing the vast majority of cases, still has not been satisfactorily addressed. The development of therapies for dry AMD has been limited, in part, by the lack of consistent models of localized retinal degeneration. ${ }^{2} \mathrm{~A}$ wide variety of genetic alterations have been discovered or engineered in rodents, ${ }^{3-5}$ dogs,${ }^{6}$ cats,${ }^{7}$ and pigs, ${ }^{8}$ which induce global retinal degeneration similar to retinitis pigmentosa (RP). These approaches affect the whole retina, and most of the proposed genetic models of AMD develop slowly, requiring many months and even years ${ }^{7}$ before reaching the stage of significant decrease in visual functions.
\end{abstract}

Faster and spatially localized retinal degeneration has been attempted using photodamage, ${ }^{5,9-13}$ which can be induced via photochemical, photothermal, or photomechanical mechanisms. Photochemical lesions typically are induced in albino animals by long exposures to bright light, leading to formation of free radicals in the photoreceptors and RPE, ${ }^{14-17}$ though the precise mechanism of photoreceptor cell death is unknown. This approach has been used to selectively eliminate photoreceptors in rats. ${ }^{10}$ However, the extensive use of ultraviolet (UV) light also can induce cataracts due to strong absorption of short wavelengths in the lens. ${ }^{18}$ The use of visible light $(450<\lambda<$ $700 \mathrm{~nm}$ ) to create photothermal damage in the outer retina helps avoiding cataract formation. In laser photocoagulation of the retina, the laser light is absorbed primarily by melanin in highly pigmented cells of the RPE and choroid. With pulses shorter than $50 \mu \mathrm{s}$, tissue damage is produced primarily by mechanical disruption of the RPE due to explosive vaporization of melanosomes. ${ }^{19}$ Microsecond-scale exposures also can be produced using rapidly-scanning continuous laser beam, which has been used for selective destruction of the RPE cells. ${ }^{20}$ However, RPE cells migrate from the adjacent untreated areas, restoring continuity of the RPE in the damage zone within 
days. ${ }^{11,20}$ Therefore, this approach did not help creating a durable model of local loss of RPE. Longer pulses can destroy tissue by denaturation of the biomolecules during hyperthermia. Milliseconds-long pulses can affect not only the RPE, but also photoreceptors and even inner retina due to heat diffusion from the RPE. ${ }^{21,22}$ Creating large $(>200 \mu \mathrm{m})$ areas of uniform and selective damage to photoreceptors has been difficult, since the heat diffusion from a large spot affects the inner retina as well. ${ }^{21,22}$ On the other hand, small lesions have been shown to contract and heal over time due to photoreceptor migration, ${ }^{12,20,23}$ and, therefore, cannot provide a stable model of local retinal degeneration. ${ }^{24}$ Instead of a single large spot, one could sequentially apply a pattern of multiple spots to cover a large area. ${ }^{25}$ Very often, however, areas between the laser spots are underexposed, resulting in islands of the functional retina in the treated zone.

We applied a high power laser scanning to coagulate not only RPE, but also photoreceptors, while preserving the inner retina. Confluent scanning provides a uniform coverage of the large zone $(2 \times 2 \mathrm{~mm})$ to prevent significant contraction of the lesion, and thereby create a stable model of localized retinal degeneration.

While photoreceptor degeneration and subsequent retinal plasticity has been observed in chronic retinal detachment, ${ }^{26-29}$ another way to create local photoreceptor degeneration was serendipitously discovered when the first subretinal implants were placed in normally-sighted animals. ${ }^{30,31}$ The chronic separation of the photoreceptors from RPE and from choroidal perfusion was shown to trigger a complete loss of the photoreceptors above the implant, while preserving the inner retina. This model is appealing because the lesion area and timing of the degeneration are well-defined, and this model can be used even in albino animals. However, in animal species with avascular retina, such as rabbits, the retinal supply of oxygen and nutrients depends entirely on diffusion from the choroid. Therefore, separation of the retina from the choroid by an impermeable subretinal implant causes atrophy of all retinal layers in a course of a few weeks.

We assessed the dynamics of photoreceptor degeneration and analyzed its effects on inner retinal neurons using these two procedures.

\section{Materials AND Methods}

\section{Subretinal Surgeries}

Nonpermeable plastic (SU-8 2025, permanent epoxy negative photoresist; Microchem, Inc., Westborough, MA, USA) disks 1 $\mathrm{mm}$ in diameter and $30 \mu \mathrm{m}$ in thickness were produced lithographically, and coated with parylene-N for biocompatibility. All animal studies were conducted in accordance with the institutional guidelines at Stanford, and conformed to the Statement for the Use of Animals in Ophthalmic and Vision research of the Association for Research in Vision and Ophthalmology (ARVO).

Wild type (WT, $n=13$ ) Long-Evans adult rats from Charles River Farm (Wilmington, MA, USA) were housed with a 12hour light/12-hour dark cycle, with food and water ad libitum. Rats with retinal degeneration were obtained from a Royal College of Surgeons (RCS, $n=5$ ) colony maintained at the Stanford Animal facility.

For surgeries, animals (P35-P50) were anesthetized with ketamine $(75 \mathrm{mg} / \mathrm{kg}$ ) and Xylazine $(5 \mathrm{mg} / \mathrm{kg})$, delivered by intramuscular injection. Subretinal implantations were performed as previously described. ${ }^{31}$ Briefly, the sclera and choroid were incised to create a retinal detachment using saline solution, and the implant was inserted into the subretinal space. The incision then was sutured and treated with a local antibiotic (bacitracin/polymyxin B).

Fluorescein angiography (FA) and optical coherence tomography (OCT; Spectralis; Heidelberg Engineering, Heidelberg, Germany) were used to assess retinal condition in the treated area in vivo.

\section{Photocoagulation With a Scanning Laser}

Dutch Belted rabbits (5-6 months old, weight 2-3 kg, $n=7$ ) were used in accordance with the ARVO Statement Regarding the Use of Animals in Ophthalmic and Vision Research after approval from the Stanford University Animal Institutional Review Board. The rabbits were anesthetized using ketamine hydrochloride $(35 \mathrm{mg} / \mathrm{kg}$, intramuscular [IM]), xylazine $(5 \mathrm{mg} /$ $\mathrm{kg}, \mathrm{IM})$, and glycopyrrolate $(0.1 \mathrm{mg} / \mathrm{kg}, \mathrm{IM})$ administered 15 minutes before the procedure.

Pupillary dilation was achieved by 1 drop each of $1 \%$ tropicamide and $2.5 \%$ phenylephrine hydrochloride. Topical tetracaine $0.5 \%$ was instilled in the eye before the treatment.

The lesions were produced by applying a continuous scanning laser (PASCAL, $532 \mathrm{~nm}$ wavelength, with custom software; Topcon, Tokyo, Japan) over a pattern of $2 \times 2 \mathrm{~mm}$ square, using a $100 \mu \mathrm{m}$ diameter spot moving at $1.6 \mathrm{~m} / \mathrm{s}$ velocity, at a power level of $2 \mathrm{~W}$. This configuration produced $60 \mu$ s exposures on the retina. With a circular flying spot, the edges of the irradiated lines experience shorter exposures, and, therefore, reduced damage, compared to its center. To improve uniformity of the tissue exposure, the adjacent lines in the scanning pattern were overlapping by 0.25 beam diameter, that is, the next line in the pattern was shifted by $75 \mu \mathrm{m}$ relative to the previous one. To account for inadvertent eye movements, which sometimes result in gaps in the tissue coverage, we applied the same scanning pattern twice over the same area, with a $100 \mathrm{~ms}$ delay between the scanning patterns. Each rabbit eye contained 1-day, 1-week, and 1-, 2-, and 3month lesions.

\section{Histology and Immunohistochemistry}

Rat eyes with SU8 implants were enucleated after $2(n=3), 5$ $(n=3)$, and $12(n=3)$ weeks for WT rats, and 12 weeks only for RCS rats $(n=3)$. Rabbit eyes $(n=6)$ were enucleated 3 months after the first laser treatment, and they contained 1-day, 1-week, and 1-, 2-, and 3-month lesions. All eyes were fixed in $1.25 \%$ or $2.5 \%$ glutaraldehyde, $1 \%$ paraformaldehyde fixative prepared in $0.1 \mathrm{M}$ sodium cacodylate buffer with $5 \mathrm{mM}$ calcium chloride and 5\% sucrose for 24 hours at room temperature. Lenses were removed and eyes were trimmed to a block size and postfixed in $2 \%$ aqueous osmium tetroxide for 2 hours at room temperature. Tissue then was dehydrated in graded alcohol, infiltrated with propylene oxide and epoxy (Araldite/Embed; Electron Microscopy Sciences, Hatfield, PA, USA), embedded in pure epoxy, and polymerized at $60^{\circ} \mathrm{C}$ for 24 hours. Thin sections $(1 \mu \mathrm{m})$ were taken (Ultracut E; Leica, Deerfield, IL, USA), stained with $0.5 \%$ toluidine blue, and slides were examined under a light microscope.

Computational molecular phenotyping (CMP) was performed on rat retina implanted for 4 months $(n=2)$. Neurons were labeled for CMP using an array of small molecule signatures (L-asparate, L-glutamate, glycine, L-glutamine, glutathione, Y-aminobutyric acid [GABA], taurine) as described previously. ${ }^{32}$ Briefly, eyes were enucleated, retina was resected, dehydrated in graded methanol and acetone, and embedded in epoxy resin for sectioning at $200 \mathrm{~nm}$ onto 12-spot Teflon-coated slides (Cell-Line; Thermo-Fisher Scientific, Waltham, MA, USA). Antibody exposure and silver intensification were performed using a silver intensification protocol de- 

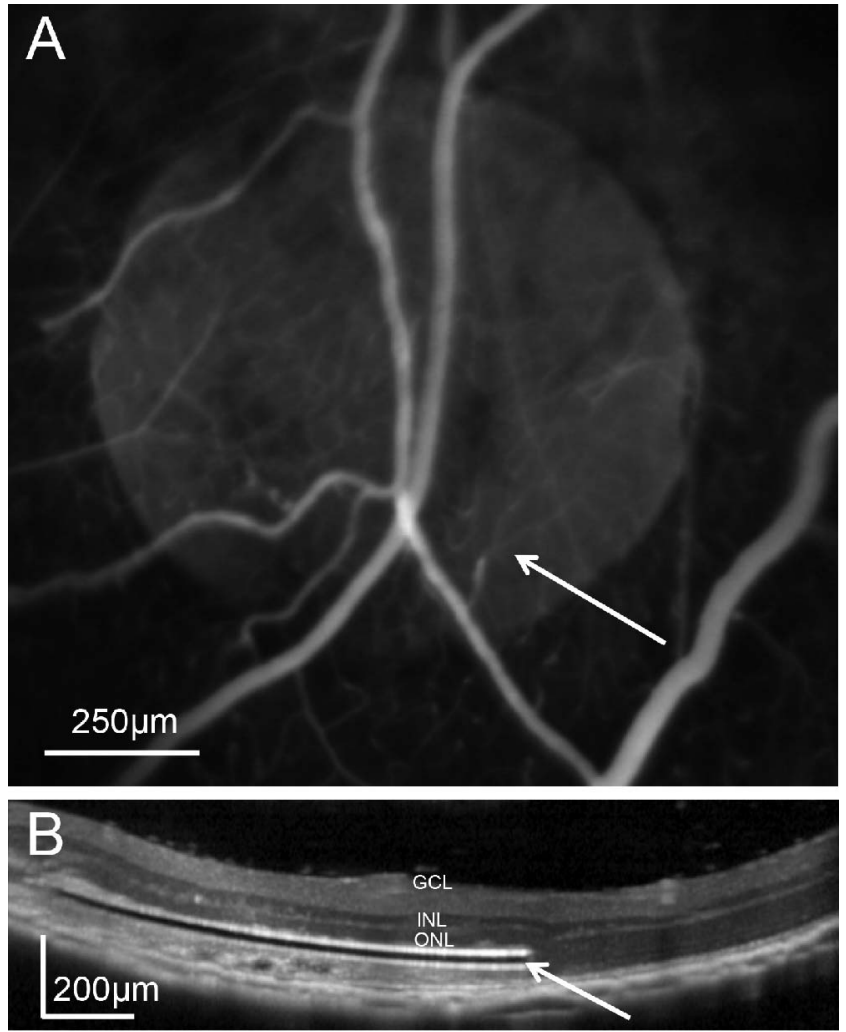

Figure 1. In vivo assessment of subretinal implantation. (A) Fluorescein angiography of the implanted rat eye reveals normal retinal blood perfusion above the subretinal implant (white arrow). (B) Optical coherence tomography of the retina above the $30-\mu \mathrm{m}$ thick subretinal implant (white arrow) 1 week after surgery.

scribed previously. ${ }^{33}$ Incubation of all antibodies generated against small molecular targets was performed overnight at room temperature and visualized with goat anti-rabbit secondary IgG coated with $1 \mathrm{~nm}$ gold (Jackson ImmunoResearch, West Grove, PA, USA) and silver intensified. Adobe Photoshop CS6 (Adobe Systems, San Jose, CA, USA) was used for final image generation.

For flat mount immunohistochemistry, retinas from WT ( $n=$ 2) and RCS $(n=2)$ rats surrounding the implant were gently separated from the sclera and immediately placed in $4 \%$ paraformaldehyde, $\mathrm{pH} \mathrm{7.4,} \mathrm{for} 60$ minutes at room temperature. Retina pieces were washed for at least 30 minutes, 6 times, in a modified PBS solution $(0.1 \% \mathrm{NaN} 3,0.5 \%$ Triton $\mathrm{X}$ 100) at room temperature before each incubation period. The tissue was blocked for 2 days in modified PBS with 3\% donkey serum at $4{ }^{\circ} \mathrm{C}$. The tissue was incubated in primary and then secondary antibodies for 5 and 2 days, respectively, with $1 \%$ donkey serum in modified PBS at $4^{\circ} \mathrm{C}$. Retinas were again washed before being mounted onto slides, with 4'6-diamidino2-phenylindole (DAPI, 1:5000 from $1 \mathrm{mg} / 1 \mathrm{~mL}$ starting concentration) added during the fourth 30-minute wash. We used the following primary antibodies: rabbit anti-PKC $\alpha$ (1:100; Santa Cruz Biotechnology, Inc., Dallas, TX, USA) and goat anti-ChAT (1:100; Millipore, Billerca, MA, USA). The following secondary antibodies (1:1000; Life Technologies, Frederic, MD, USA) were paired with the appropriate primary antibodies: donkey anti-goat $555 \mathrm{~nm}$ and donkey anti-rabbit $647 \mathrm{~nm}$. Retinas were mounted ganglion cell side up onto a glass slide with Vectashield (H-1000; Vector Laboratories, Burlingame, CA, USA) and imaged on a Leica confocal microscope with $\times 40$ or $\times 63$ objectives. ImageJ (available in the public domain at http://imagej.nih.gov/ij/) was used to generate Z-projections and cross sections from Z-stacks.

\section{Cell Counting and Thickness Measurements}

For cell counting, light microscopy pictures $(\times 20)$ at the center of the lesions were acquired. Similar images of the control tissue were taken at least $500 \mu \mathrm{m}$ away from the edge of the lesion. Cells in the outer (ONL) and inner (INL) nuclear layers were counted in one picture per lesion, and the entire number of nuclei was counted. For the ganglion cell counting, three different sections per lesion were used, and the number of cells averaged. Thickness of the retinal layers was measured at the center of the lesions.

All measurements were expressed as the ratio between the treated and control areas to avoid disparities in retinal location $(n=3 \mathrm{WT}$ and $n=3 \mathrm{RCS})$.

\section{Scanning Electron Microscopy (SEM)}

For SEM, four eyes from three rabbits were enucleated rapidly; the anterior segment of the globe was incised and separated from the posterior part of the eyecup; and retina was peeled from the RPE. In one animal, the eyecup was incubated for 1 hour in a calcium/magnesium-free PBS to facilitate retinal detachment and increase RPE integrity. The remaining sclera, choroid, and RPE were fixed in glutaraldehyde-paraformaldehyde fixative $(1.25 \% / 1 \%$ in $0.2 \mathrm{M}$ sodium cacodylate buffer) at room temperature for 30 minutes. The tissue then was fixed for an additional 18 hours at $48^{\circ} \mathrm{C}$, rinsed in a buffer, and postfixed in $1 \%$ osmium tetroxide in $0.2 \mathrm{M}$ sodium cacodylate, pH 7.4. The tissue was washed with distilled water, dehydrated in a series of ethanols, transferred to absolute ethanol, and critical point dried. The dried tissues then were mounted on stubs, plasma coated with gold/palladium (Denton Vacuum, Moorestown, NJ, USA), and imaged with a Hitachi S-34000N VP-SEM (Hitachi, Placeton, CA, USA).

\section{Live/Dead Fluorescent Assay}

Immediately after laser treatment, the RPE cells were fused to the photoreceptors outer segments, and, therefore, the apical side of the RPE cannot be imaged with SEM. To evaluate viability of these cells, we performed live/dead staining of the retina and fused RPE cells $(n=1)$. The tissue was immersed in $2 \mu \mathrm{M}$ Calcein AM/4 $\mu \mathrm{M}$ EthD-III (Viability/Cytotoxicity Assay; Biotium, Hayward, CA, USA) solution for 30 minutes. Tissue samples were promptly imaged with fluorescence microscopy.

\section{Results}

\section{Degeneration of Photoreceptors Above the Subretinal Implant}

One week after implantation of the SU8 sheets in rats (see Methods), FA revealed normal blood perfusion above the implant (Fig. 1A), and OCT demonstrated that the retina was reattached in the implanted area (Fig. 1B). However, thinning of the outer retina already was noticeable in OCT even after 1 week.

Histology at 2, 5, and 12 weeks after implantation revealed rapid loss of photoreceptors above the implant, and excellent preservation of the retina just outside the implanted area (Fig. 2). The number of photoreceptor nuclei decreased over time (Fig. 3): at 2 weeks, the outer segments of photoreceptors were lost, but many of the nuclei in the ONL still preserved 

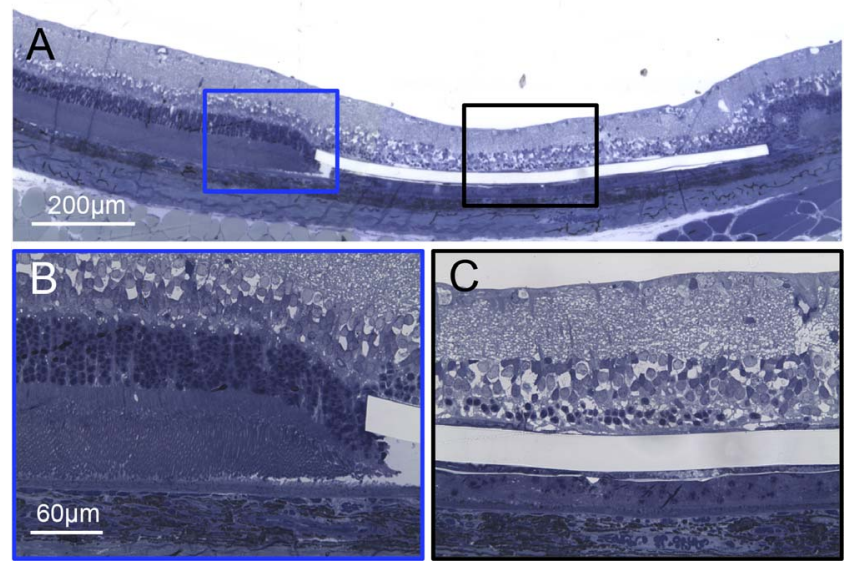

FiguRE 2. Implanted retina at 5 weeks after surgery. (A) Wide view of the retina demonstrates highly localized degeneration zone above the implant. (B) Higher magnification of the normal retina at the very edge of the implant. (C) Above the implant, most of the photoreceptors, except for a few nuclei, are gone, while the inner retinal layers remain apparently preserved.

(Fig. 3B). At 5 weeks, the vast majority of the ONL was lost as well (Fig. 3C). At 12 weeks after implantation, all photoreceptor nuclei had disappeared, leaving the cells in the INL in close proximity to the implant (Fig. 3D).

The rate of loss of the photoreceptor nuclei and decrease in ONL thickness and preservation of the inner retina are summarized in Figures $3 \mathrm{G}$ and $3 \mathrm{H}$. The number of photoreceptor nuclei dropped by $43 \%(P<0.05)$ after 2 weeks of implantation, and only $4 \%(P<0.001)$ survived at 12 weeks
(Fig. 3G). However, the number of nuclei and thickness of the INL and ganglion cell layer (GCL) were not significantly affected by the implant ( $P>0.05$, paired $t$-test).

Additionally, we checked whether the subretinal implantation affects retinal degeneration in a genetic rodent model the RCS rats. At 3 months, photoreceptors were lost completely in the control areas and above the implant, while the number of the inner retinal neurons remained unaffected (Figs. 3E, 3F).

\section{Inner Retinal Changes}

Although the number of cells in the INL and GCL were not affected by the implant, there were more subtle signs of remodeling and degeneration of the inner retinal network. Computational Molecular Phenotyping (see Methods) with $\operatorname{GABA}(\gamma)$, glycine $(\mathrm{G})$, and glutamate $(\mathrm{E})$ labeling $3,4,32,34$ revealed good preservation of all classes of the inner retinal cells at 4 months (Fig. $4 \mathrm{~A}$, compared to $4 \mathrm{~B}$ ), but also initiation of microneuromas formation with appearance of swellings indicative of ectopic synapses (Fig. 4C, arrows). Additional staining with taurine $(\tau)$, and glutamine $(Q)$ and glutamate $(E)$ ( $\tau$ GE) did not show any significant Müller cell hypertrophy over the implanted area (data not shown).

The laminar structure of the retina above the implant was assessed by staining starburst amacrine cells with choline acetyl transferase (ChAT). The laminar structure of the starburst amacrine cells in the implanted area was very similar to the normal control (Figs. 5A, 5B), indicating no major disruptions of the inner retinal network at 3 months after surgery.

However, immunohistochemical labeling of the rod bipolar cell terminals with PKC antibodies revealed abnormalities in the shape of the terminals 3 months after implantation. Unlike
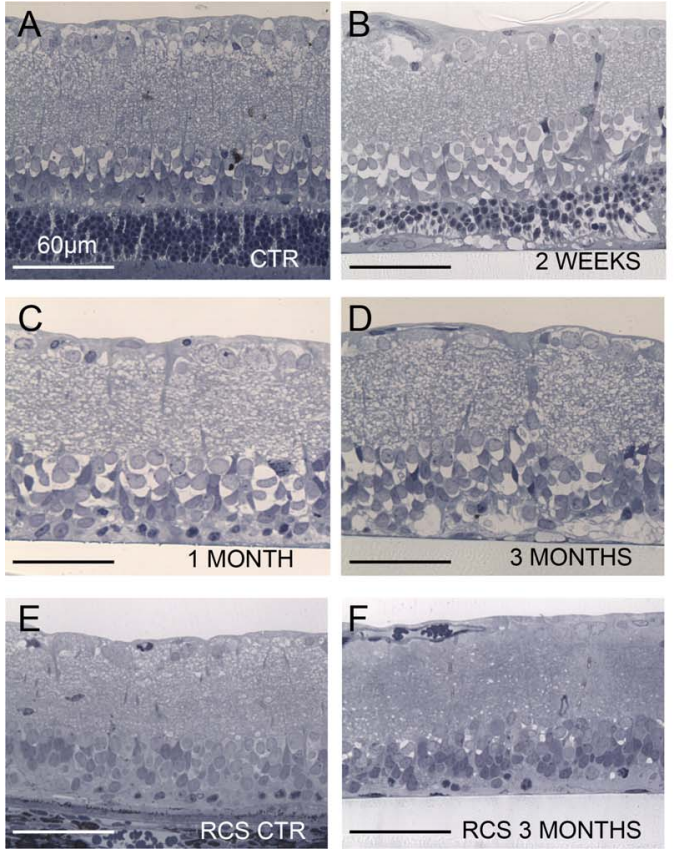

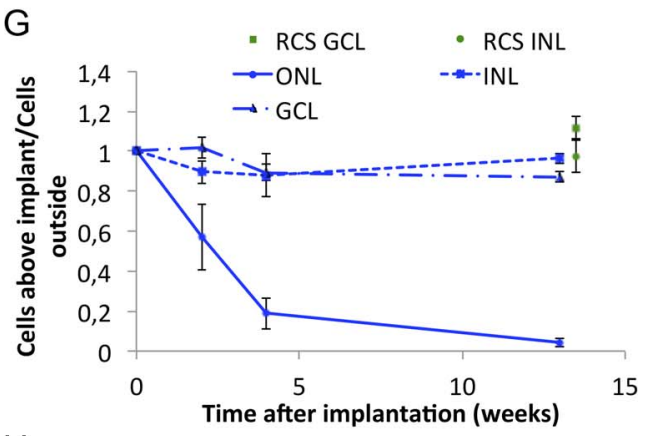

$\mathrm{H}$

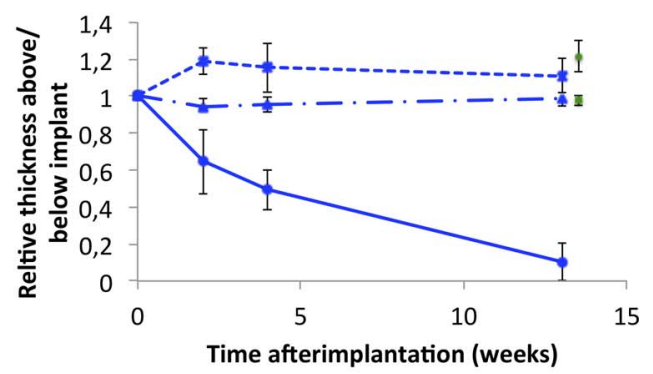

Figure 3. Dynamics of retinal degeneration. (A) Control retina outside the implanted area. (B-D) Retina above the implant 2 weeks, 1 month, and 3 months after surgery, respectively, illustrating the progressive loss of PR cell bodies and stability of the inner retina. (E, F) The RCS retina outside of the implanted area and above the implant 3 months after surgery. The subretinal implantation does not seem to affect the RCS retina. (G) Number of nuclei in various layers above the implant over time. The number of photoreceptor nuclei (ONL) decrease to $4 \%$ at 12 weeks after surgery, while the GCL and INL numbers remain similar to control. Similarly, implantation in RCS rats does not affect the number of cells in these layers. (H) Relative thickness of the retinal layers above the implant compared to control area. The photoreceptor layer thickness follows similar decrease reflecting the loss of nuclei, whereas the GCL thickness remains stable. Interestingly, the INL slightly widens 2 weeks after implantation, and then returns to the baseline level over time. 

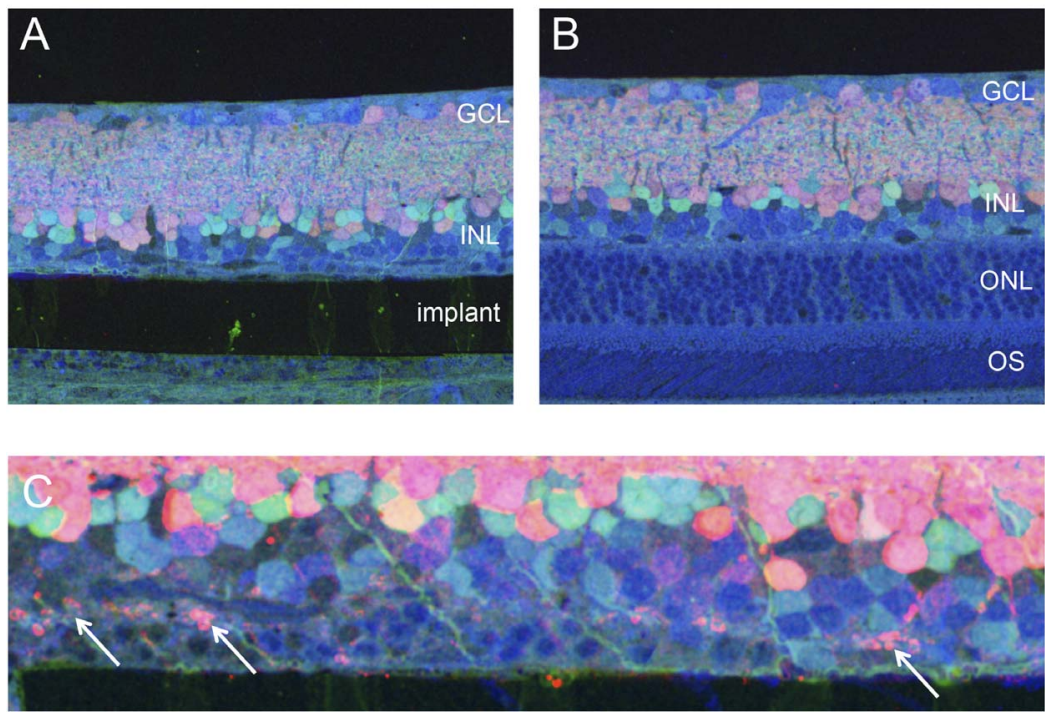

Figure 4. Molecular phenotyping of the implanted retina. (A, B) Implanted and control areas labeled with GABA (red), glycine (green), and glutamate (blue) revealing the cell types and dendrites. (C) Higher magnification reveals ectopic synapses (white arrows) as signs of degeneration in the outer plexiform layer.

the large and round normal axonal terminals (Figs. 6A, 6B), the terminals in the implanted region were much smaller and had irregular morphology (Figs. 6C, 6D), similar to the 6-month-old RCS rats without an implant (Figs. 6E, 6F). This change in morphology of the synapses in the inner plexiform layer above the implant is most likely due to local deprivation of presynaptic signals onto bipolar cells and has been observed before in different models of retinal degeneration. ${ }^{35,36}$

\section{Retinal Degeneration in Laser Lesions}

Chronic separation of the retina from RPE and choroid in avascular species, such as rabbits, leads to a complete degeneration of all retinal layers. Therefore, instead of using a subretinal implant, in such species the photoreceptors can be eliminated by selective photocoagulation (see Methods).

One hour after application of the scanning laser, timeresolved FA demonstrated damage to the blood-retinal barrier in the treatment zone (Fig. 7A; Supplementary Fig. S1) appearing 10 seconds after injection, when fluorescein starts diffusing from the choriocapillaris. Damage to the RPE cells in this area was confirmed by a positive dead staining of the RPE cells over the lesioned area (Fig. 7C). However, already 1 week after the treatment the FA pattern appeared in the earlier phase of the FA - right after the injection and did not change over time (Fig. 7C and Supplementary Fig. S1), indicating that this was not due to fluorescein diffusion into the retina, but rather due to reduced pigmentation of the RPE in and around the treatment zone (so called window defect). This change demonstrates restoration of the blood retinal barrier integrity. Scanning electron microscopy of the lesion 1 week after treatment showed migration of RPE cells from the surrounding area (Figs. 7D, 7E).

Histology also demonstrated damage to photoreceptors at 1 day (Fig. 8), with shortened outer segments, damaged inner segments, and pyknotic photoreceptor nuclei, while the inner retina remained intact (Figs. 8A, 8B). One week after treatment, photoreceptors largely disappeared, while cells in the inner retina remained intact (Fig. 8C). At this stage, the RPE layer displayed a drastic decrease in pigmentation. From 1 to 3 months after laser application, the RPE cells in the treated area increased in size and regained their pigment, while photore- ceptors remained absent, and the number of the inner retinal neurons unchanged (Figs. 8D, 8F). Dynamics of the photoreceptors loss and preservation of the INL is summarized in Figure 8G.

\section{Discussion}

Establishment of reliable and well-controlled animal models of local retinal degeneration is important not only for developing therapies for geographic atrophy in AMD, but also for better understanding the changes in the inner retina associated with the loss of photoreceptors.

Selective ablation of RPE cells alone by laser does not result in a model of geographic atrophy due to rapid migration of the RPE cells from adjacent areas and restoration of the RPE monolayer, even after multiple treatments. ${ }^{11}$ We decided to slow down the laser scanning speed to damage the photoreceptors directly as well, thereby creating millimeters-wide and uniform area of RPE and photoreceptor damage. Since photoreceptors do not migrate over distances larger than approximately $100 \mu \mathrm{m},{ }^{20,23,37}$ these scotomata remain permanent despite the repopulation of the treated area by RPE cells.
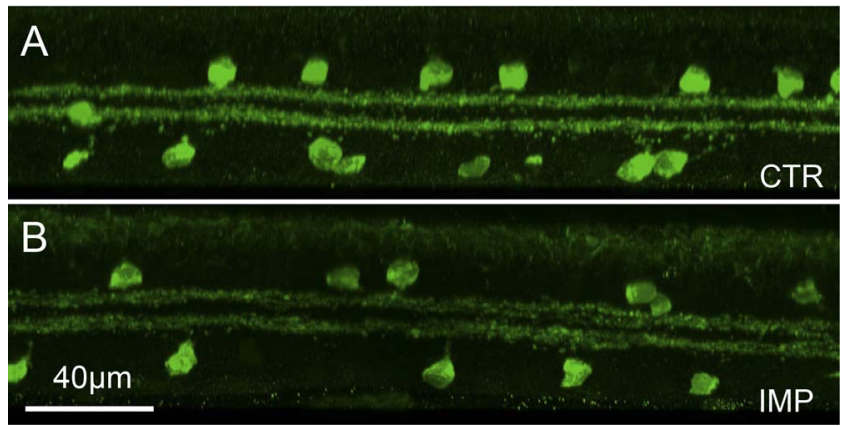

Figure 5. Amacrine cells stratification. (A, B) Control and implanted regions labeled with ChAT antibodies. Starburst amacrine cells expressing ChAT stratify at a very specific depth in the inner plexiform layer in the ON and OFF strata. Stratification in the implanted area at 3 months is very similar to intact retina. 


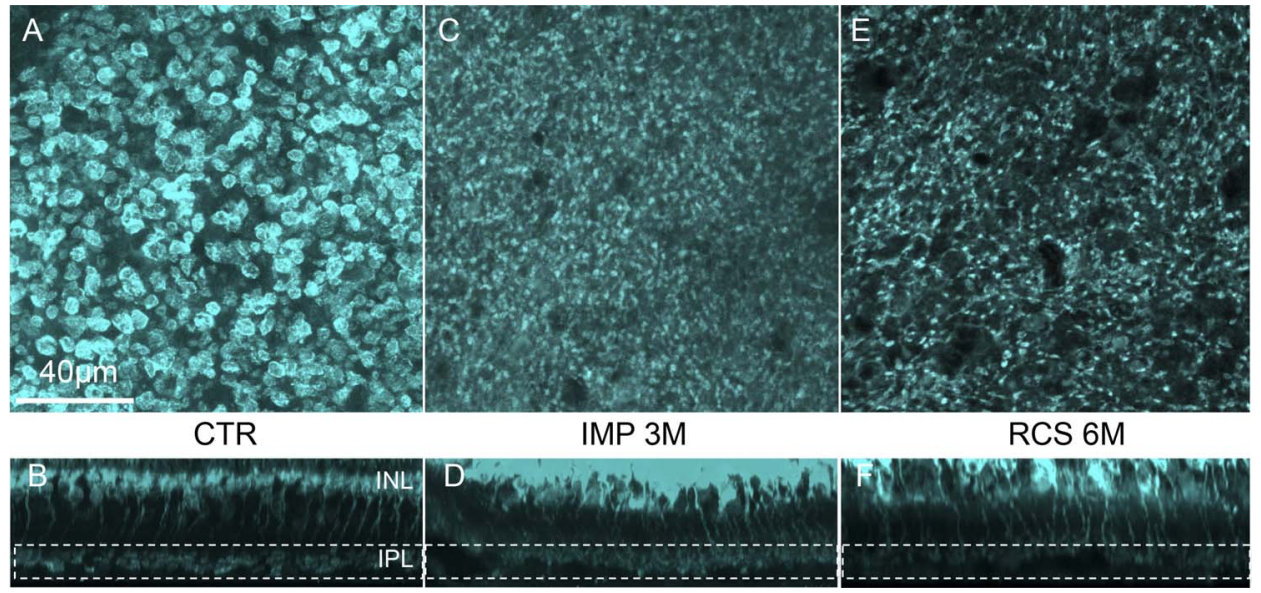

Figure 6. Rod bipolar cells structure. (A, B) Confocal planar and cross-sectional view of the rod bipolar cell terminals in normal retina (away from the implant). (C, D) Similar staining above the implant reveals greatly reduced size and irregular shape of the axon terminals. (E, F) The RCS rat retina has similarly abnormal shape and size of the bipolar cell terminals.

Preservation of the inner retina in this model was made possible by relatively short $(60 \mu \mathrm{s})$ exposure time, which limited the heat diffusion deeper into the retina.

Since laser light is absorbed primarily by melanin, the laser model of retinal damage is limited to pigmented animals. Subretinal implantation provides an alternative approach applicable to nonpigmented species. It creates a similarly uniform and selective degeneration of photoreceptors while preserving the inner retinal neurons. However, the survival of the retinal cells separated from RPE and choroid relies on the nutrients delivered via retinal vasculature. Therefore, this subretinal implant model can be used only in species with vascular retinas. Together, these two complementary approaches can be used in a wide variety of animal species. Both of them trigger a uniform and well-defined local degeneration of photoreceptor cells, while leaving the inner retina intact, as summarized in the Table.

It is important to emphasize though, that the models reported here do not reproduce the time course and pathophysiology of dry AMD initiated by degeneration of the RPE, but rather reproduce the ultimate outcome of the disease characterized by localized degeneration of photoreceptors, surrounded by normal retina. In both models, RPE cells survived or migrated back inside the treated area.

Preservation of the inner retina after photoreceptors degeneration and spatial localization of the scotoma are two important features to consider in rehabilitation strategies. The loss of photoreceptor cells decreases the input to postsynaptic bipolar and ganglion cells, but may not eliminate such signals completely, unlike the global forms of degeneration induced by genetic mechanisms. Indeed, lateral interactions via horizontal or amacrine cells with broad dendritic arbors might continue providing stimulation to the inner retinal neurons, perhaps maintaining the retinal network in a partially active state and decreasing the extent of its restructuring compared to global degeneration. ${ }^{3,4}$ In human patients, this is manifested by better preservation of the inner retina above the areas of geographic atrophy, compared to end-stage RP. ${ }^{38-41}$ In addition, genetic manipulations often have diverse consequences, making it difficult to isolate contribution of one particular mechanism, such as the loss of photoreceptors, from others.

Although, after photoreceptors death, the functional integrity of the inner retinal network cannot be probed with conventional light stimuli, electrical stimulation of bipolar cells with subretinal implants has already demonstrated preservation of some aspects of the retinal signal processing, such as flicker fusion and adaptation to constant illumination, ${ }^{42}$ which are emerging from the inner retinal network. This preservation of the inner retinal circuitry is beneficial for meaningful reintroduction of the visual information using optical ${ }^{43-46}$ or electrical ${ }^{31,47-49}$ stimulation.

Our models, therefore, provide a convenient way to study the effect of the lesion size and duration on the extent of the inner retinal rewiring. It also would be very interesting to see whether the retinal network remains plastic and rewires constructively when meaningful inputs are reintroduced either by artificial stimulation or by transplantation of cells replacing the lost photoreceptors. The size and shape of the bipolar cell terminals might be a very convenient indicator of these changes. In the absence of the neural input, these terminals decrease in size and lose their spherical shape (Fig. 5). An important question, therefore, is whether or not chronic stimulation of bipolar cells would restore their normal morphology.

Additionally, local models of retinal degeneration provide an opportunity to study interactions of normal and prosthetic vision - a likely combination for the majority of patients. Indeed, emerging strategies of optical stimulation, such as optogenetics ${ }^{44,50}$ and photoswitches ${ }^{45,46}$ affect the degenerate and normal retinas differently, and interaction of these two pathways should be studied in the appropriate animal models before reaching the clinical stage. For example, the healthy peripheral retina and artificial stimulation over the degenerated central region might affect each other by adaptation effects.

TABLE. Summary of the Features for Two Methods Producing Local Loss of Photoreceptors

\begin{tabular}{lccccc}
\hline & $\begin{array}{c}\text { Selective PR } \\
\text { Degeneration }\end{array}$ & $\begin{array}{c}\text { Preservation of } \\
\text { the Inner Retina }\end{array}$ & Reversibility & $\begin{array}{c}\text { Species } \\
\text { Requirements }\end{array}$ \\
\hline Subretinal implantation & Yes & Yes & Yes & Retinal vasculature & Invasive: sclerotomy, retinal detachment \\
Laser treatment & Yes & Yes & No & Pigmentation & Noninvasive \\
\hline
\end{tabular}



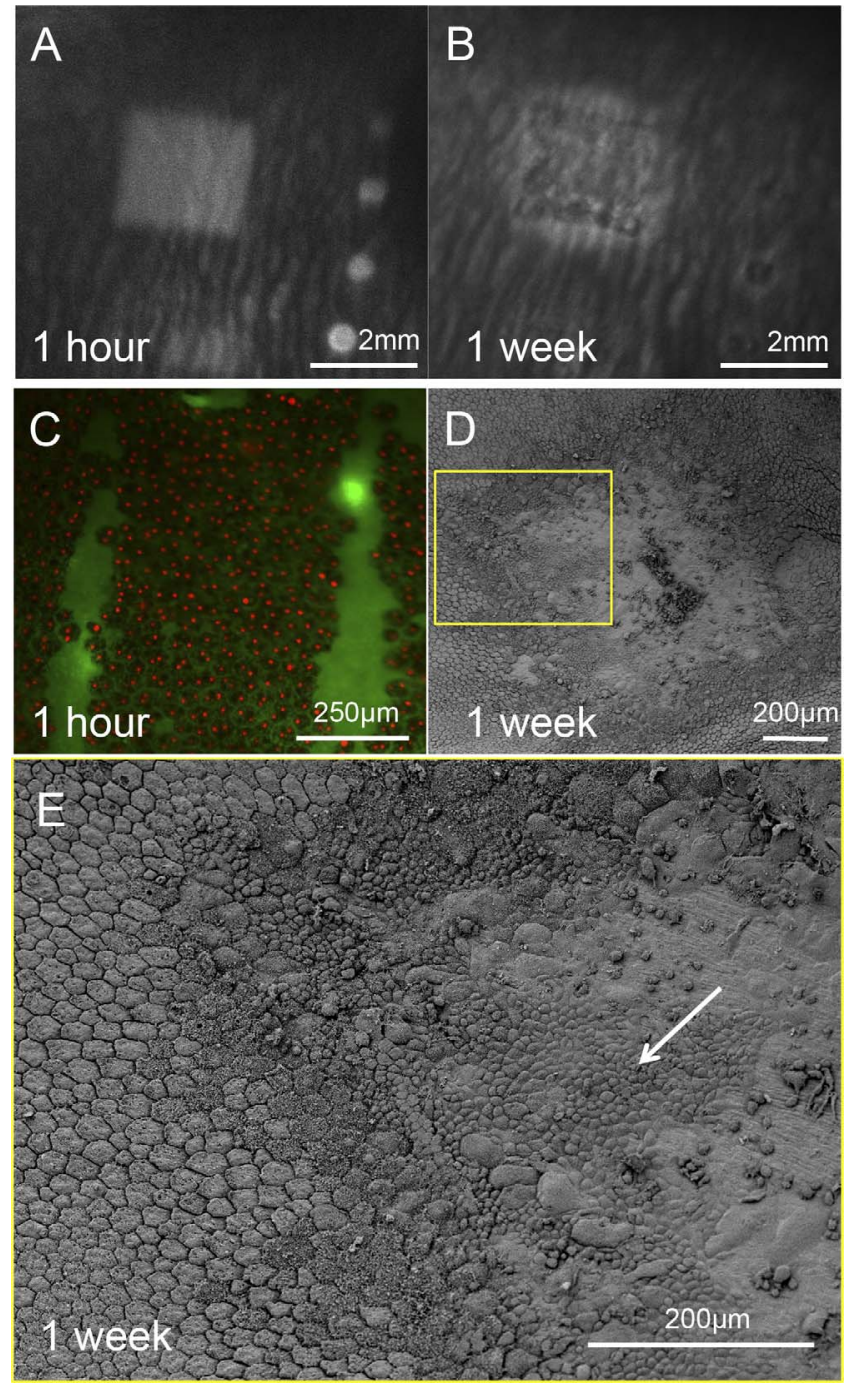

Figure 7. Rabbit retina after acute laser lesion. (A) Late phase FA (4 minutes after injection) of the rabbit fundus 1 hour after laser application reveals the $2 \times 2 \mathrm{~mm}$ damage zone. This pattern started to appear 10 seconds after the fluorescence in the choroid suggesting a leakage through the blood-retinal barrier. (B) Early phase FA of the same lesion 1 week after the treatment demonstrates lighter pigmentation of the RPE inside and adjacent to the treatment zone. No changes of the image in the late phase FA suggest restoration of the blood-retinal barrier. (C) Fluorescent assay of a lesion 1 hour after laser treatment demonstrates that all RPE cells in the treatment zone are dead. (D, E) Scanning electron microscopy of the RPE 1 week after laser application demonstrates initial restoration of the RPE monolayer in the lesion by migrating cells from the untreated adjacent area (white arrow).

Normal and artificial vision in the same retina may have different properties, such as different flicker fusion frequencies. It will be important to evaluate such interactions in electrophysiological and behavioral experiments, and ideally, optimize the stimulation parameters to enable fusion of the two modalities.

Finally, explantability of subretinal implants, as performed by Retina Implant $A G$ in humans, ${ }^{51,52}$ or the use of biodegradable implants ${ }^{53}$ provides an interesting opportunity to study restoration of the photoreceptor outer segments and associated changes in the retinal network. These studies also can help define the timeline for reversible damage to
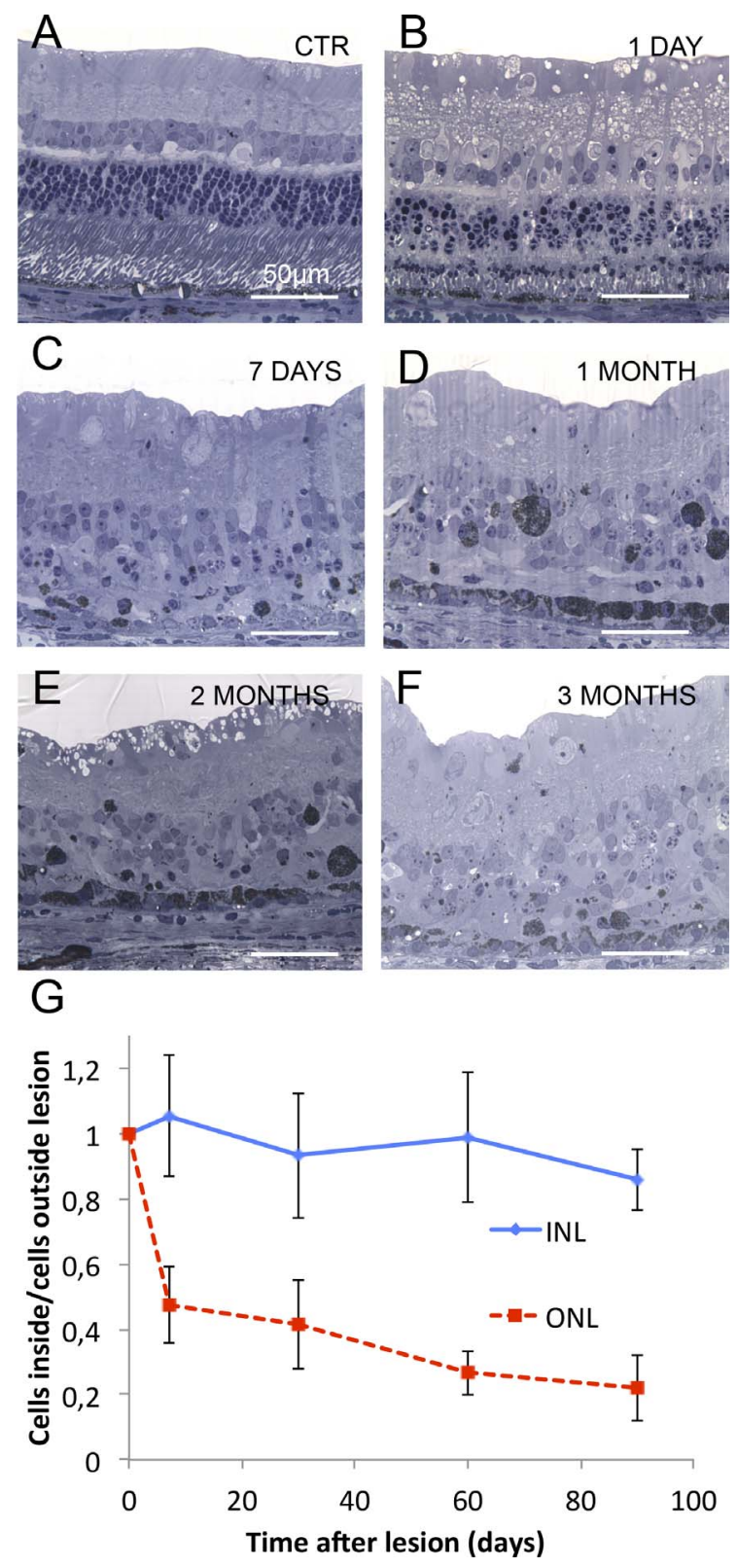

Figure 8. Dynamics of retinal degeneration after laser treatment. (AF) Rabbit retina in the untreated control area (A), and at different time points (1 day, 1 week, 1 month, 2 months, and 3 months) after treatment (B-F). Most of the photoreceptor nuclei are already lost by 1 week, and the rest slowly disappear over the 3-month follow-up period. Although the inner retina appears wavy, the number of cells in the inner retinal layers seems to be preserved even at late stages. (G) Number of cells in ONL and INL over time after lasering. Unlike with subretinal implants, approximately $20 \%$ of the ONL cells are still present at 3 months.

photoreceptors during chronic retinal detachment and assess the success of strategies, such as photoreceptor transplantation, ${ }^{54}$ into the damaged area for treatment of retinal degeneration.

\section{Acknowledgments}

The authors thank Michael Marmor, MD, and George Goetz, PhD, for fruitful discussions and support, as well as Xin Lei, MSc, and Ludwig Galambos, MSc, for implant fabrication. 
Supported by the National Institutes of Health (NIH; Grant R01-EY018608), the Department of Defense (Grant W81XWH-15-1-0009), NIH CTSA (Award UL1 RR025744), the Stanford Spectrum fund, and Pew Charitable Trusts Scholarship in the Biomedical Sciences (AS); and by the Foundation Voir et Entendre (Paris) and Pixium Vision (HL).

Disclosure: H. Lorach, None; J. Kung, None; C. Beier, None; Y. Mandel, None; R. Dalal, None; P. Huie, None; J. Wang, None; $\mathbf{S}$. Lee, None; A. Sher, None; B.W. Jones, None; D. Palanker, None

\section{References}

1. Wong WL, Su X, Li X, et al. Global prevalence of age-related macular degeneration and disease burden projection for 2020 and 2040: a systematic review and meta-analysis. Lancet Glob Health. 2014;2:e106-e116.

2. Zeiss CJ. Animals as models of age-related macular degeneration: an imperfect measure of the truth. Vet Pathol. 2010;47: 396-413.

3. Marc RE, Jones BW, Watt CB, Strettoi E. Neural remodeling in retinal degeneration. Prog Retin Eye Res. 2003;22:607-655.

4. Jones BW, Marc RE. Retinal remodeling during retinal degeneration. Exp Eye Res. 2005;81:123-137.

5. Marc RE, Jones BW, Watt CB, Vazquez-Chona F, Vaughan DK, Organisciak DT. Extreme retinal remodeling triggered by light damage: implications for age related macular degeneration. Mol Vis. 2008;14:782-806.

6. Beltran WA. The use of canine models of inherited retinal degeneration to test novel therapeutic approaches. Vet Ophthalmol. 2009;12:192-204.

7. Narfstrom K. Progressive retinal atrophy in the Abyssinian cat. Clinical characteristics. Invest Ophthalmol Vis Sci. 1985;26: 193-200.

8. Ross JW, Fernandez de Castro JP, Zhao J, et al. Generation of an inbred miniature pig model of retinitis pigmentosa. Invest Ophthalmol Vis Sci. 2012;53:501-507.

9. Youssef PN, Sheibani N, Albert DM. Retinal light toxicity. Eye. 2011;25:1-14.

10. Busch EM, Gorgels TG, van Norren D. Temporal sequence of changes in rat retina after UV-A and blue light exposure. Vision Res. 1999;39:1233-1247.

11. Lavinsky D, Chalberg TW, Mandel Y, et al. Modulation of transgene expression in retinal gene therapy by selective laser treatment. Invest Ophthalmol Vis Sci. 2013;54:1873-1880.

12. Strazzeri JM, Hunter JJ, Masella BD, et al. Focal damage to macaque photoreceptors produces persistent visual loss. Exp Eye Res. 2014;119:88-96.

13. Vogel A, Capon MR, Asiyo-Vogel MN, Birngruber R. Intraocular photodisruption with picosecond and nanosecond laser pulses: tissue effects in cornea, lens, and retina. Invest Ophthalmol Vis Sci. 1994;35:3032-3044.

14. Glickman RD. The origin of photo-oxidative stress in the aging eye. Prog Brain Res. 2001;131:699-712.

15. Verma L, Venkatesh P, Tewari HK. Phototoxic retinopathy. Opbthalmol Clin N Am. 2001;14:601-609.

16. Solley WA, Sternberg P Jr. Retinal phototoxicity. Int Ophthalmol Clin. 1999;39:1-12.

17. Arnault E, Barrau C, Nanteau C, et al. Phototoxic action spectrum on a retinal pigment epithelium model of age-related macular degeneration exposed to sunlight normalized conditions. PLoS One. 2013;8:e71398.

18. Michael R. Development and repair of cataract induced by ultraviolet radiation. Ophthal Res. 2000;32(suppl 1):1-44.

19. Schuele G, Rumohr M, Huettmann G, Brinkmann R. RPE damage thresholds and mechanisms for laser exposure in the microsecond-to-millisecond time regimen. Invest Ophthalmol Vis Sci. 2005;46:714-719.
20. Paulus YM, Jain A, Nomoto H, et al. Selective retinal therapy with microsecond exposures using a continuous line scanning laser. Retina. 2011;31:380-388.

21. Sramek C, Paulus Y, Nomoto H, Huie P, Brown J, Palanker D. Dynamics of retinal photocoagulation and rupture. J Biomed Opt. 2009;14:034007.

22. Jain A, Blumenkranz MS, Paulus Y, et al. Effect of pulse duration on size and character of the lesion in retinal photocoagulation. Arch Ophthalmol. 2008;126:78-85.

23. Sher A, Jones BW, Huie $\mathrm{P}$, et al. Restoration of retinal structure and function after selective photocoagulation. J Neurosci. 2013;33:6800-6808.

24. Lavinsky D, Cardillo JA, Mandel Y, et al. Restoration of retinal morphology and residual scarring after photocoagulation. Acta Ophthalmol. 2013;91:e315-e323.

25. Blumenkranz MS, Yellachich D, Andersen DE, et al. Semiautomated patterned scanning laser for retinal photocoagulation. Retina. 2006;26:370-376.

26. Fisher SK, Lewis GP, Linberg KA, Barawid E, Verardo MR. Cellular remodeling in mammalian retina induced by retinal detachment. In: Kolb H, Fernandez E, Nelson R, eds. Webvision: The Organization of the Retina and Visual System. Salt Lake City, UT: University of Utah Health Sciences Center; 1995.

27. Fisher SK, Lewis GP. Muller cell and neuronal remodeling in retinal detachment and reattachment and their potential consequences for visual recovery: a review and reconsideration of recent data. Vision Res. 2003;43:887-897.

28. Marc RE, Murry RF, Fisher SK, Linberg KA, Lewis GP. Amino acid signatures in the detached cat retina. Invest Ophthalmol Vis Sci. 1998;39:1694-1702.

29. Marc RE, Murry RF, Fisher SK, Linberg KA, Lewis GP, Kalloniatis M. Amino acid signatures in the normal cat retina. Invest Ophthalmol Vis Sci. 1998;39:1685-1693.

30. Pardue MT, Stubbs EB Jr, Perlman JI, Narfstrom K, Chow AY, Peachey NS. Immunohistochemical studies of the retina following long-term implantation with subretinal microphotodiode arrays. Exp Eye Res. 2001;73:333-343.

31. Mandel Y, Goetz G, Lavinsky D, et al. Cortical responses elicited by photovoltaic subretinal prostheses exhibit similarities to visually evoked potentials. Nat Commun. 2013;4:1980.

32. Marc RE, Jones BW. Molecular phenotyping of retinal ganglion cells. J Neurosci. 2002;22:413-427.

33. Kalloniatis M, Fletcher EL. Immunocytochemical localization of the amino acid neurotransmitters in the chicken retina. $J$ Comp Neurol. 1993;336:174-193.

34. Jones BW, Watt CB, Frederick JM, et al. Retinal remodeling triggered by photoreceptor degenerations. J Comp Neurol. 2003;464:1-16.

35. Claes E, Seeliger M, Michalakis S, Biel M, Humphries P, Haverkamp S. Morphological characterization of the retina of the CNGA3(-/-)Rho(-/-) mutant mouse lacking functional cones and rods. Invest Ophthalmol Vis Sci. 2004;45:2039-2048.

36. Cuenca N, Pinilla I, Sauve Y, Lund R. Early changes in synaptic connectivity following progressive photoreceptor degeneration in RCS rats. Eur J Neurosci. 2005;22:1057-1072.

37. Paulus YM, Jain A, Gariano RF, et al. Healing of retinal photocoagulation lesions. Invest Ophthalmol Vis Sci. 2008;49: 5540-5545.

38. Green WR, Enger C. Age-related macular degeneration histopathologic studies. The 1992 Lorenz E. Zimmerman Lecture. Ophthalmology. 1993;100:1519-1535.

39. Bird AC, Phillips RL, Hageman GS. Geographic atrophy: a histopathological assessment. JAMA Ophthalmol. 2014;132: 338-345.

40. Jones BW, Kondo M, Terasaki H, Lin Y, McCall M, Marc RE. Retinal remodeling. Jpn J Ophthalmol. 2012;56:289-306. 
41. Sullivan RK, Woldemussie E, Pow DV. Dendritic and synaptic plasticity of neurons in the human age-related macular degeneration retina. Invest Opbthalmol Vis Sci. 2007;48: 2782-2791.

42. Lorach H, Goetz G, Mandel Y, et al. Performance of photovoltaic arrays in-vivo and characteristics of prosthetic vision in animals with retinal degeneration. Vision Res. 2014.

43. Busskamp V, Duebel J, Balya D, et al. Genetic reactivation of cone photoreceptors restores visual responses in retinitis pigmentosa. Science. 2010;329:413-417.

44. Busskamp V, Picaud S, Sahel JA, Roska B. Optogenetic therapy for retinitis pigmentosa. Gene Ther. 2012;19:169-175.

45. Polosukhina A, Litt J, Tochitsky I, et al. Photochemical restoration of visual responses in blind mice. Neuron. 2012; 75:271-282.

46. Tochitsky I, Polosukhina A, Degtyar VE, et al. Restoring visual function to blind mice with a photoswitch that exploits electrophysiological remodeling of retinal ganglion cells. Neuron. 2014;81:800-813.

47. Mathieson K, Loudin J, Goetz G, et al. Photovoltaic retinal prosthesis with high pixel density. Nat Photonics. 2012;6: 391-397.
48. Zrenner E, Bartz-Schmidt KU, Benav H, et al. Subretinal electronic chips allow blind patients to read letters and combine them to words. Proc Biol Sci. 2011;278:1489-1497.

49. Humayun MS, Dorn JD, da Cruz L, et al. Interim results from the International Trial of Second Sight's Visual Prosthesis. Ophthalmology. 2012;119:779-788.

50. Busskamp V, Roska B. Optogenetic approaches to restoring visual function in retinitis pigmentosa. Curr Opin Neurobiol. 2011;21:942-946.

51. Stingl K, Bartz-Schmidt KU, Besch D, et al. Artificial vision with wirelessly powered subretinal electronic implant alpha-IMS. Proc Biol Sci. 2013;280:20130077.

52. Stingl K, Bartz-Schmidt K-U, Gekeler F, Kusnyerik A, Sachs H, Zrenner E. Functional outcome in subretinal electronic implants depends on foveal eccentricity. Invest Ophthalmol Vis Sci. 2013;54:7658-7665.

53. Lyu S, Untereker D. Degradability of polymers for implantable biomedical devices. Int J Mol Sci. 2009;10:4033-4065.

54. Pearson RA, Barber AC, Rizzi M, et al. Restoration of vision after transplantation of photoreceptors. Nature. 2012;485:99103. 